\title{
Calidad de vida y función sexual en mujeres postmenopáusicas con incontinencia urinaria
}

\author{
Herrera Pérez A*, Arriagada Hernández J**, González Espinoza C**, Leppe Zamora J***, \\ Herrera Neira $\mathrm{F}^{* * * *}$.
}

*Geriatra, Médico Internista. Clínica Las Condes. Facultad de Medicina Universidad de Chile.

**Kinesiólogo. Escuela de Kinesiología, Facultad de Medicina Universidad de Chile.

***Kinesiólogo. Unidad de Investigación Clínica y PBE, Escuela de Kinesiología. Universidad de Chile.

****Kinesiólogo. Escuela de Kinesiología. Universidad de Chile. Universidad Católica del Maule, Chile.

Actas Urol Esp. 2008;32(6):624-628

\section{RESUMEN}

CALIDAD DE VIDA Y FUNCIÓN SEXUAL EN MUJERES POSTMENOPÁUSICAS CON INCONTINENCIA URINARIA

Introducción: El envejecimiento constituye un proceso natural e inevitable, se manifiesta mediante cambios físicos, psicológicos, emocionales, sociales y que puede provocar eventos indeseados, como la Incontinencia Urinaria (IU). Tal condición se incrementa con la edad, es más frecuente en la mujer y puede producir trastornos en la calidad de vida, como aislamiento social, temor, vergüenza, postergación personal, etc.

El presente estudio tuvo por objetivo, describir la calidad de vida y la función sexual en mujeres posmenopáusicas según el tipo de IU a través del uso de cuestionarios específicos.

Material y Método: Estudio descriptivo, transversal, entre junio y septiembre del 2006, participaron 46 mujeres postmenopáusicas mayores de 50 años, con diagnóstico clínico y urodinámico de IU, vida sexual activa en los últimos 3 meses. Todas las participantes contestaron el Cuestionario de Salud King (KHQ) y Î́ndice de Función Sexual Femenina (IFSF).

Resultados: De acuerdo al tipo de IU se encontró que el 43,5\% presentó IU Mixta, el 37\% IU Esfuerzo y el 19,6\% IU Urgencia.

Los dominios más afectados en el total de las mujeres con respecto a calidad de vida fue "impacto de la IU" (65,2\%), "actividades realizadas" (56,5\%) y el menos afectado fue "relaciones personales" $(15,2 \%)$. La IU Mixta $(43,5 \%)$ tuvo mayor afectación en la mayoría de los dominios del $\mathrm{KHQ}$, lo que indica mayor deterioro de calidad de vida.

Los desórdenes más prevalentes de la función sexual femenina fueron la disminución del "deseo" $(45,7 \%)$ y "excitación” (30,4\%) y los dominios menos afectados fueron la "satisfacción" $(8,7 \%)$ y "dolor" $(6,5 \%)$. El tipo de IU que presentó los puntajes más bajos en el IFSF fue la IU Mixta.

Conclusiones: La IU afecta la calidad de vida y la función sexual siendo la IUM la que presenta mayor grado de afectación, en comparación a los otros dos tipos.

Debido a que la IU es una condición altamente tratable, es necesaria una evaluación integral de estos pacientes, bajo un modelo biopsico-social, teniendo como paradigma central, mejorar la calidad de vida, considerando la propia percepción del paciente.

Palabras clave: Incontinencia urinaria. Incontinencia urinaria esfuerzo. Incontinencia urinaria urgencia. Calidad de vida. Función sexual. Postmenopausia.

\section{ABSTRACT}

GUALITY OF LIFE AND SEXUAL FUNCTION IN POSTMENOPAUSAL WOMEN WITH URINARY INCONTINENCE

Introduction: Aging is a natural unavoidable process that is expressed through physical, psychological, emotional, and social changes, and that can produce undesirable events, such as Urinary Incontinence (UI). This condition increases with age, is more frequent in women, and can produce impairments in the quality of life, such as social isolation, fear, embarrassment, and self-postponement, among others. This research aimed at describing the quality of life and sexual function in postmenopausal women according to the UI classification through the application of specific questionnaires.

Material and Method: This is a descriptive and cross-sectional study carried out between June and September, 2006. This study included 46 postmenopausal women over 50 years old with clinical and urodynamic diagnosis of UI, active sexual life in the last 3 months. All the subjects answered the King's Health Questionnaire (KHQ) and Female Sexual Function Index (FSFI).

Results: According to the UI classification, 43.5\% showed Mixed UI, 37\% Stress UI, and 19.6\% Urge UI. The most affected domains in the total of the subjects in regards to Quality of Life were "UI impact" (65.2\%), "activities performed" (56.5\%), and the less affected domain was "personal relations" (15.2\%). The most affected UI classification in the KHQ domains was Mixed UI (43.5\%), which indicates a greater impairment of the quality of life. The most prevalent disorders of the female sexual function were: decrease of "sexual desire" (45.7\%) and "excitation" (30.4\%), and the less affected domains were "satisfaction" $(8.7 \%)$ and "pain" $(6.5 \%)$. The classification of UI that showed the lower punctuations in FSFI was the Mixed UI.

Conclusion: UI affects quality of life and sexual function being the mixed UI the classification that showed the greatest influence compared to the other two types. Due to the UI is a highly-treatable condition, a comprehensive assessment of these patients is needed, under a bio-psycho-social model, having as central paradigm the improvement of the quality of life, considering the perception of the patients themselves

Keywords: Urinary incontinence. Stress. Urge, Quality of life. Sexuality. Postmenopausical. 
$\mathrm{L}$ a Incontinencia Urinaria (IU), según la International Continence Society (ICS) se define como "cualquier pérdida involuntaria de orina" ${ }^{1}$.

Estimaciones señalan que, la IU afecta entre el $3 \%$ hasta el 55\% dependiendo de la definición de incontinencia usada y la edad de la población estudiada.

A pesar que la IU puede causar aislamiento social, disminución de la función sexual y otros problemas psicológicos ${ }^{2,3}$, permanece con frecuencia como un problema oculto, ya que sólo un tercio de los que la sufren busca ayuda profesional, lo que tiene un impacto psicosocial y en la calidad de vida de las personas.

Las mujeres sexualmente activas relatan que la IU puede alterar su función sexual de distintas maneras, como baja en la líbido, miedo a la pérdida de orina durante el acto sexual y depresión ${ }^{4,5}$.

Actualmente, esta condición indeseada es foco de una demanda significativa de programas sociales y discusión frecuente entre los sistemas de salud sobre la mejor prevención y terapéutica ${ }^{6}$.

En Chile no existen estudios determinantes acerca de la prevalencia de la IU, y tampoco existen datos sobre el estado en que se encuentran los pacientes, lo que dificulta la toma de medidas que vayan en su beneficio. Por este motivo es necesario el uso de instrumentos específicos que permitan evaluar el impacto de la IU en diferentes poblaciones tanto desde el punto de vista global como específico, como es el caso de la investigación de la sexualidad.

El presente estudio tiene por objetivo evaluar la calidad de vida y la función sexual de mujeres posmenopáusicas según los distintos tipos de incontinencia urinaria, antes de recibir cualquier tipo de tratamiento, así como también despertar conciencia sobre la necesidad de considerar a la mujer que envejece, como un todo aunado a su ambiente, apoyados en la búsqueda de causas corregibles de los problemas que, tratados en su momento evitaran la discapacidad, dependencia y empeoramiento de calidad de vida a futuro.

\section{PACIENTES Y MÉTODO}

El estudio fue del tipo descriptivo, no experimental, transversal. Se llevó a cabo entre los meses de Junio y Septiembre del 2006, en las Unidades de Climaterio de una clínica privada, de Santiago de Chile.
La población de estudio quedó definida según los siguientes criterios de inclusión: sexo femenino, postmenopáusicas, mayores de 50 años, diagnóstico clínico y urodinámico de incontinencia urinaria, sin tratamiento actual, vida sexual activa en los últimos 3 meses y participación voluntaria en el estudio. Criterio de exclusión: enfermedad neurológica invalidante, cáncer urogenital y/o cirugía mutilante, patologías psiquiátricas y/o alteración cognitivo avanzado.

Los sujetos de estudio fueron reclutados por los investigadores según los criterios de inclusión/ exclusión y con el consentimiento informado de cada uno de los pacientes.

La obtención de los datos se realizó inmediatamente después de la atención médica. Se les entregó los Cuestionario de Salud King (KHQ) y el Índice de Función Sexual Femenina (IFSF), los que fueron autoadministrados y devueltos inmediatamente a los investigadores. Ambos cuestionarios fueron realizados, antes de iniciar cualquier tipo de tratamiento (farmacológico, quirúrgico y/o kinésiológico) para la IU.

El Cuestionario de Salud King (KHQ), evalúa la calidad de vida en pacientes con IU, fue clasificado como grado A según la International Consultation on Incontinence (ICI), pues ha cumplido con las exigencias internacionales de este tipo de instrumentos, siendo ampliamente reconocido y utilizado ${ }^{7}$.

Fue diseñado para ser autoadministrado. Consta de 21 ítems distribuidos en 9 dimensiones: percepción del estado de salud general ( 1 ítem); impacto de la IU ( 1 ítem); limitación de roles (2 ítems); limitaciones sociales (2 items); limitaciones físicas (2 ítems); relaciones personales (3 ítems); emociones (3 ítems); sueño/energía (2 ítems), y el impacto de la incontinencia (5 ítems). Una dimensión adicional evalúa la gravedad de los síntomas urinarios. Cada ítem del KHQ tiene una escala de respuesta tipo Likert con 4 posibles opciones. El porcentaje de afectación en cada dimensión va de 0\% (mejor calidad de vida) a $100 \%$ (peor calidad de vida). El puntaje total fluctúa entre un mínimo de 28 puntos y un máximo de 115 puntos. A mayor puntaje total obtenido mayor influencia de la IU en la calidad de vida $^{8,9}$.

El segundo cuestionario es el Índice de Función Sexual Femenina, consta de 19 preguntas y se agrupa en seis dominios: deseo, excitación, lubricación, 
orgasmo, satisfacción y dolor; cada pregunta tiene 5 ó 6 opciones, asignándoles un puntaje que va de 0 a 5. El puntaje de cada dominio se multiplica por un factor y el resultado final es la suma aritmética de los dominios. El puntaje total es entre 0 a 36 puntos, siendo a mayor puntaje mejor sexualidad ${ }^{10,11}$.

\section{Análisis Estadístico}

Se utilizó el programa SPSS 13.0. Se implementaron las pruebas de Kruskall-Wallis y Wilcoxon para establecer diferencias significativas entre gru$\operatorname{pos}(\alpha=0,05)$.

\section{RESULTADOS}

La muestra fue constituida por 46 mujeres. La mediana de la edad fue de 56 años; con un límite inferior de 50 y un límite superior de 73 años. De acuerdo al tipo de IU se encontró que el 43,5\% presentó IUM, el 37\% IUE y el 19,6\% IUU.

\section{Cuestionario de Salud King (KHQ)}

En relación al puntaje total del KHQ según el tipo de IU se observó que la IUE presentó una mediana de 41 puntos, la IUU 57 puntos y la IUM 56 puntos (Fig. 1).

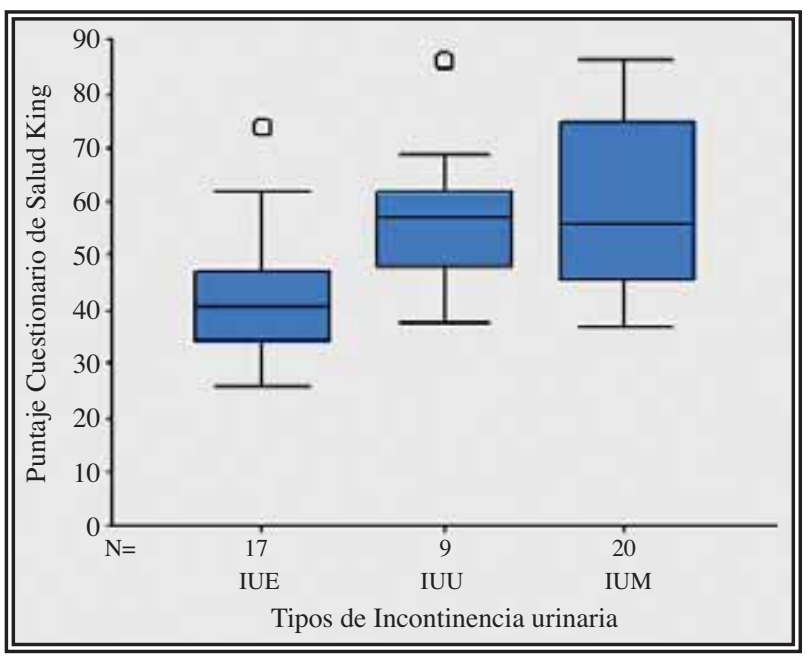

FIGURA 1. Medianas puntaje total $\mathrm{KHQ}$ según tipo de IU.

Se encontraron diferencias estadísticamente significativas $(\mathrm{p}<0,05)$ entre la IUE/IUU, lo mismo ocurrió entre la IUE/IUM, lo que no sucedió entre la IUU/IUM.

\section{Dominios KHQ según tipo IU}

Lo sucedido en los distintos dominios se puede observar en la Tabla 1.
Entre la IUE/IUU se observaron diferencias estadísticamente significativas en los dominios de "impacto en su vida" y "emociones" ( $<<0,05)$.

Entre la IUE/IUM estas diferencias se encontraron en los dominios "percepción de mujer", "impacto en su vida", "limitación de roles", "limitación física", "limitación social" y "sueño/energía". No se encontraron diferencia significativas entre los dominios de la IUU y IUM ( $p>0,05)$.

Con respecto al dominio "impacto de la IU en su vida” se observó que la IUM fue la que presentó mayor grado de afectación (Fig. 2).

\section{Índice de Función Sexual Femenina (IFSF)}

De acuerdo a puntajes totales del IFSF según tipo de IU se encontró una mediana de 27,2 puntos para la IUE; 27,6 puntos para la IUU y 23.6 puntos para la IUM (Fig. 3).

No existieron diferencias estadísticamente significativas entre los distintos tipos de IU (p>0,05).

\section{Dominios IFSF según tipo IU}

Lo sucedido en los diversos dominios se puede observar en la Tabla 2.

Se observaron diferencias significativas entre la IUE/IUU en el dominio "dolor" y entre la IUE/IUM en los dominios de "dolor" y "orgasmo" $(p<0,05)$. Entre la IUU/IUM no se observaron tales diferencias.

\section{DISCUSIÓN}

En el presente estudio se encontró que el mayor porcentaje de mujeres presentó IUM $(43,5 \%)$. Este tipo de IU presentó afectación superior en la mayoría de los dominios del $\mathrm{KHQ}$, excepto en "percepción de la mujer", "emociones” y "actividades realizadas”, que fue superada por la IUU.

El tipo de IU que presentó los puntajes más bajos en el IFSF fue la IUM.

Si bien es cierto, la IU por sí misma, es capaz de producir un deterioro en la calidad de vida, se encontró que las mujeres con IUM, tuvieron una afectación mayor, siguiéndole en importancia la IUU.

La mayoría de los estudios publicados en los últimos años, coinciden que estos dos tipos de IU son los más afectados, produciendo incomodidad en grado similar, indicando que es el síntoma de urgencia, el que produce un mayor deterioro en la calidad 
Tabla 1. Medianas de dominios del KHQ según tipo de IU.

\begin{tabular}{lccc}
\hline \multicolumn{4}{c}{ RIQ: Rango intercuartílico } \\
\hline Dominios KHQ & $\begin{array}{c}\text { Mediana IUE } \\
\text { (RIQ) (n=17) }\end{array}$ & $\begin{array}{c}\text { Mediana IUU } \\
\text { (RIQ) (n=39) }\end{array}$ & $\begin{array}{c}\text { Mediana IUM } \\
\text { (RIQ) (n=20) }\end{array}$ \\
\hline Percepción de la mujer & $25(0-37,5)$ & $50(25-62,5)$ & $50(25-50)$ \\
Impacto IU en su vida & $33,3(33,3-66,7)$ & $66,7(66,7-100)$ & $83,3(41,7-100)$ \\
Limitaciones de roles & $16,7(0-33,3)$ & $16,7(0-58,3)$ & $58,3(33,3-91,7)$ \\
Limitación física & $16,7(0-50)$ & $16,7(8,3-74,8)$ & $50(16,7-66,7)$ \\
Limitación social & $0(0-11,1)$ & $0(0-66,7)$ & $22,2(0-50)$ \\
Rel. Personales & $0(0-0)$ & $0(0-16,7)$ & $0(0-50)$ \\
Emociones & $11,1(0-27,8)$ & $33,3(16,7-94,4)$ & $27,8(0-41,7)$ \\
Sueño y energía & $0(0-25)$ & $16,7(0-58,3)$ & $33,3(16,7-50)$ \\
Act. Realizadas & $50(28,8-75)$ & $75(33,3-91,7)$ & $54,1(33,3-75)$ \\
\hline
\end{tabular}

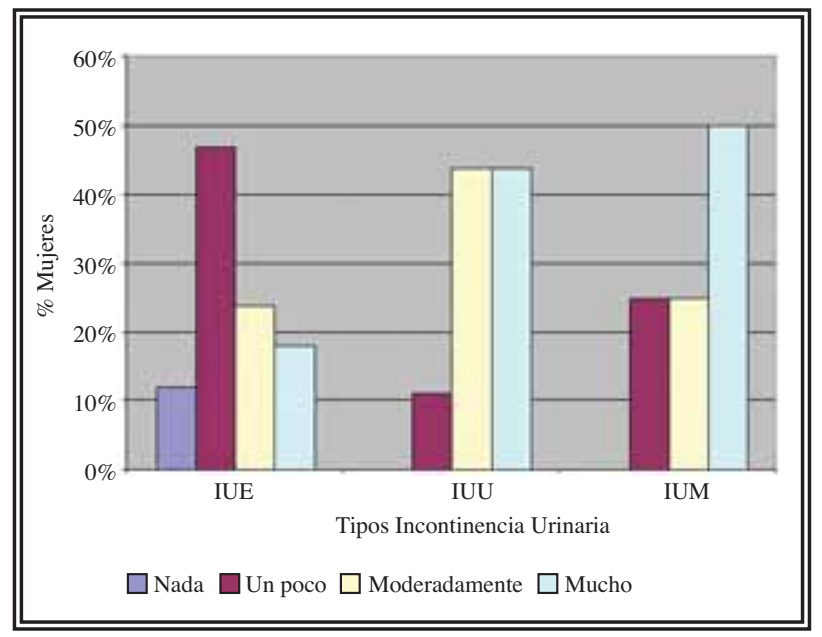

FIGURA 2. Dominio "Impacto de IU en su vida" según tipo IU.

de vida, en términos de impacto, debido a que las personas no pueden predecir su incontinencia, por no poseer control sobre su vejiga ${ }^{12,13}$.

Las mujeres con IUE presentaron un menor deterioro en su calidad de vida, ya que, serían capaces de adaptar su forma de vida para evitar situaciones que provoquen la pérdida involuntaria de

Tabla 2. Medianas de dominios del IFSF según tipo de IU

\begin{tabular}{lccc}
\hline $\begin{array}{l}\text { Dominios } \\
\text { IFSF }\end{array}$ & $\begin{array}{c}\text { Mediana IUE } \\
\text { (RIQ) (n = 17) }\end{array}$ & $\begin{array}{c}\text { Mediana IUU } \\
\text { (RIQ) (n = 9) }\end{array}$ & $\begin{array}{c}\text { Mediana IUM } \\
\text { (RIQ) (n = 20) }\end{array}$ \\
\hline Deseo & $3,6(1,5-4,5)$ & $3,6(3-4,2)$ & $3(2,4-3,6)$ \\
Excitación & $3,6(3-5,2)$ & $4,2(3,4-5,1)$ & $3,9(2,7-4,7=$ \\
Lubricación & $4,8(3,4-5,8)$ & $4,5(3-5,5)$ & $3,7(3-5,2)$ \\
Orgasmo & $4,8(3,4-5,6)$ & $3,6(3,1-5,4)$ & $3,4(2,8-4,8)$ \\
Satisfacción & $4,8(3,8-5,6)$ & $4(3,4-6)$ & $4,8(3,7-5,2)$ \\
Dolor & $6(5-6)$ & $4(3,6-5,8)$ & $4,8(3,6-5,9)$ \\
\hline
\end{tabular}

orina, por ejemplo, actividades que impliquen ejercicio físico, cargar peso, etc ${ }^{14}$. Es importante destacar que este tipo de IU, presentó menor limitación de roles, dentro y fuera del hogar, debido a que la mayoría señaló no sentirse limitadas físicamente en las tareas hogareñas, puesto que contaban con ayuda en el hogar.

La función sexual, no sólo depende de los niveles estrogénicos, sino que es una complementariedad de éstos con aspectos sicológicos, sociales, ambientales y culturales; factores que son propios de cada mujer y que le dan la característica de ser una cualidad individual ${ }^{15-17}$.

Es importante mencionar, que por el diseño del estudio no podemos atribuir causalidad ni asociación entre la IU y la función sexual. Según Tannenbaum $^{18}$ la IU no deteriora la vida sexual por sí misma, pero se asocia a un número de factores que influencian la actividad sexual, tal como la edad, la salud física y mental. Otros señalan que aunque la incontinencia tiene un efecto negativo sobre la función sexual, pocas pacientes reciben información y tratamiento especializado.

Las limitaciones del estudio indican que los resultados, son aplicables solamente a la población estudiada. Es probable la existencia de sesgos $\mathrm{u}$ omisión de información, dado la utilización de cuestionarios que tratan aspectos muy personales de la mujer.

Dado los resultados, y sabiendo que la IU es una condición altamente tratable, es necesaria una evalua- 


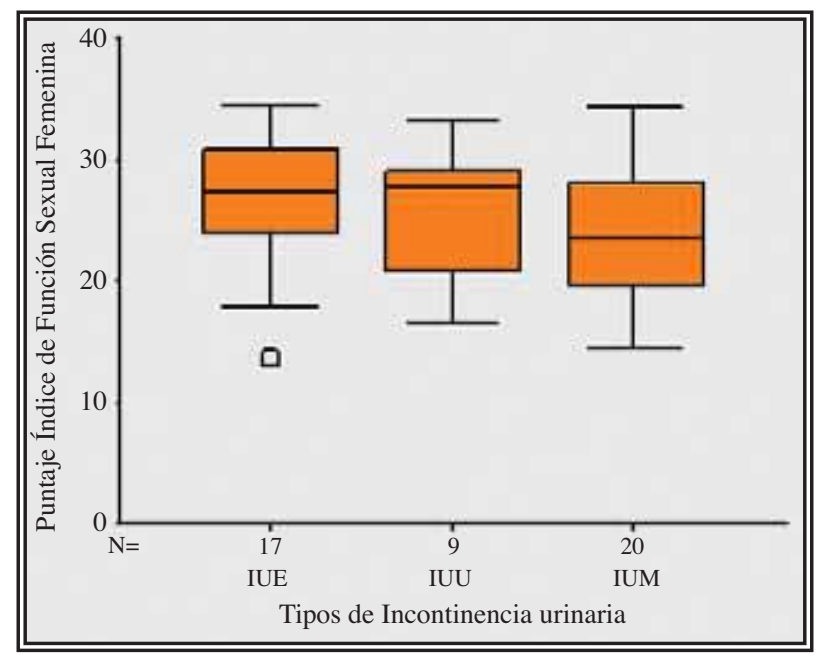

FIGURA 3. Medianas por puntaje total IFSF según tipo de IU.

ción integral de estos pacientes, bajo un modelo biopsico-social, teniendo como paradigma central, mejorar la calidad de vida.

Para concluir, quisiéramos que esta investigación permita incentivar el uso de cuestionarios en mujeres con trastornos urinarios en los Servicios de Salud, como instrumentos que permitan evaluar la intervención clínica, permitiendo considerar la propia percepción del paciente.

\section{Agradecimientos}

A las Unidades de Geriatría y Climaterio de Clínica Las Condes.

\section{REFERENCIAS}

1. Abrams P, Cardozo L, Fall M , Griffiths D, Rosier P, Ulmsten U, et al. The standardisation of terminology in lower urinary tract function. Report from the Standardisation Sub-committee of the International Continence Society. Urology 2003;61:37-49.

2. Shaw C. A review of the psychosocial predictors of help-seeking behaviour and impact on quality of life in people with urinary incontinence. J Clin Nurs. 2001:10(1):15-24.

3. Stach-Lempinen B, Hakala AL, Laippala P, Lehtinen K, Metsanoja R, Kujansuu E. Severe depression determines quality of life in urinary incontinent women. Neurourol Urodyn. 2003; 22(6):563-68.

4. Handa VL, Harvey L, Cundiff GW, Siddique SA, Kjerulff KH. Sexual function among women with urinary incontinence and pelvic organ prolapse. Am J Obst Gynecol. 2004;191(3):751-756.
5. Salonia A, Munarriz RM, Naspro R, Nappi RE, Briganti A, Chionna R, et al. Women's sexual dysfunction: a pathophysiological review. BJU Int. 2004;93(8):1156-1164.

6. Nihira MA, Henderson N. Epidemiology of urinary incontinence in women. Curr Womens Health Rep. 2003;3(4):340-347.

7. Donovan J, Bosch R. Symptoms and quality of life assessment. Edition 2005. 3rd International Consultation on Urinary Incontinence.

8. Kellegher C.J, Cardozo L, Khullar V, Salvatore S. A new questionnaire to asses the quality of life urinary incontinent women. Brit J of Obst Gynaecol. 1997;104(12):1374-1379.

9. Badía X, Castro D, Conejero J. Validez del cuestionario King`s Health Questionnaire para la evaluación de la calidad de vida de los pacientes con incontinencia urinaria. Med Clin (Barc). 2000; 114:647-652.

10. Rosen R, Brown C, Heiman J, Leiblum S, Meston C, Shabsigh $\mathrm{R}$, et al. The Female Sexual Function Index (FSFI): A Multidimensional Self-Report Instrument for the Assessment of Female Sexual Function. J Sex Marl Ther. 2000;26(2):191-208.

11. Blumel JE, Binfa L, Cataldo P, Carrasco A, Izagurre, Sarrá S. Índice de Función Sexual Femenina: un test para evaluar la sexualidad de la mujer. Revista Chilena Obstetricia y Ginecología. 2004;64(2):118-125.

12. Coyne K S, Zhou Z, Thompson C, Versi E. The impact on health-related quality of life of stress, urge and mixed urinary incontinence. BJU. 2003;92(7):731-735.

13. Simeonova Z, Milsom I, Kullendorff AM, Molander U, Bengtsson C. The prevalence of urinary incontinence and its influence on the quality of life in women from and urban Swedish population. Acta Obstet Gynecol Scand. 1999;78(6): 546-551.

14. Genazzani AR, Nicolucci A, Campagnoli C, Crosignani P, Nappi C, Serra GB, et al. Assessment of the QoL in Italian menopausal women: comparison between HRT users and non-users. Maturitas 2002;42(4):267-280.

15. Gramegna G, Blumel JE, Roncagliolo ME, Aracena B, Tacla X. Patrones de conducta sexual en mujeres chilenas. Rev Méd Chile1998;126(2):162-168.

16. Hagglund D, Walker- Engstrom M, Larsson G, Leppert J. Quality of life and seeking help in women with urinary incontinence. Acta Obstet Gynecol Scand. 2004;80(11):1051-1055.

17. Tannenbaum C, Corcos J. Assalian P. The Relationship Between Sexual Activity and Urinary Incontinence in Older Women. J Am Geriatr Soc. 2006;54(8):1220-1224.

Correspondencia autor: Dra. A. Herrera Pérez Departamento Medicina Sección Geriatría. Clínica Las Condes. Lo Fontecilla, 441 - Las Condes, Santiago, Chile Tel.: (56-2) 2104601 - (56-2) 2189148 Cel: 09-2270068 E-mail: doc.adela.herrera@gmail.com; ca gonzalez@med.uchile.cl Información artículo: Original - Urología femenina Trabajo recibido: diciembre 2007

Trabajo aceptado: abril 2008 